\title{
From structural to functional glycomics: core substitutions as molecular switches for shape and lectin affinity of $N$-glycans
}

\section{Sabine André ${ }^{1, *}$, Tibor Kožár², Shuji Kojima³, Carlo Unverzagt ${ }^{4}$ and Hans-Joachim Gabius ${ }^{1}$}

${ }^{1}$ Institute of Physiological Chemistry, Faculty of Veterinary Medicine, Ludwig Maximilians University Munich, Veterinärstr. 13, D-80539 Munich, Germany ${ }^{2}$ Institute of Experimental Physics, Department of Biophysics, Slovak Academy of Sciences, Watsonova 47, SK-04001 Košice, Slovak Republic

${ }^{3}$ Faculty of Pharmaceutical Sciences, Tokyo University of Science, Yamazaki 2641, Noda City, Chiba 278-8510, Japan

${ }^{4}$ Bioorganic Chemistry, Bldg. NW1, University of

Bayreuth, D-95440 Bayreuth, Germany

${ }^{*}$ Corresponding author

e-mail: sabine.andre@Imu.de

\begin{abstract}
Glycan epitopes of cellular glycoconjugates act as versatile biochemical signals (sugar coding). Here, we test the hypothesis that the common $\mathrm{N}$-glycan modifications by core fucosylation and introduction of the bisecting $N$ acetylglucosamine moiety have long-range effects with functional consequences. Molecular dynamics simulations indicate a shift in conformational equilibria between linear extension or backfolding of the glycan antennae upon substitution. We also present a new fingerprint-like mode of presentation for this multi-parameter system. In order to delineate definite structure-function relationships, we strategically combined chemoenzymatic synthesis with bioassaying cell binding and the distribution of radioiodinated neoglycoproteins in vivo. Of clinical relevance, tailoring the core region affects serum clearance markedly, e.g., prolonging circulation time for the neoglycoprotein presenting the $\mathrm{N}$-glycan with both substitutions. $\alpha 2,3-S i a l y l a t i o n$ is another means toward this end, similarly seen for type II branching in triantennary N-glycans. This discovery signifies that rational glycoengineering along the given lines is an attractive perspective to optimize pharmacokinetic behavior of glycosylated pharmaproteins. Of general importance for the concept of the sugar code, the presented results teach the fundamental lesson that $\mathrm{N}$-glycan core substitutions convey distinct characteristics to the concerned oligosaccharide relevant for cis and trans biorecognition processes. These modifications are thus molecular switches.
\end{abstract}

Keywords: agglutinin; drug targeting; glycoprotein; lectin; neoglycoprotein; tumor imaging.

\section{Introduction}

Posttranslational modifications are an efficient and farreaching means to extend protein functionality profoundly. Looking at the rather small covalent modifications by phosphorylation, acetylation or methylation, their impact on protein activity, the generation of modified surface epitopes sensed by distinct receptors which can subsequently initiate downstream signaling and their indexing potential underlying epigenetic processes explain why they are referred to as biological code systems (Barbieri, 2008; Sims and Reinberg, 2008). Furthermore in this respect, a distinct class of structurally much more variegated additions in protein conjugates can even 'impart a discrete recognitional role to the protein', because their building blocks are 'ideal for generating compact units with explicit informational properties', i.e., oligosaccharides (Winterburn and Phelps, 1972). Because introduction of these complex glycans matches the level of occurrence of protein phosphorylation and oligosaccharides have an unsurpassed capacity for information coding, these attributes of protein glycosylation inspired the concept of the sugar code (Gabius, 2009). It is 'the most complex known chemical code in a short sequence among all biological oligomers' (Laine, 1997) and by definition predictive of a host of downstream events. They can be triggered by two principal mechanisms: (i) altering protein folding and routing as well as reactivity with ligands or other proteins, and (ii) acting as biochemical signals decoded by lectins (Sharon and Lis, 1997; Reuter and Gabius, 1999; Solís et al., 2001; Gabius et al., 2004; Honke and Taniguchi, 2009). Hereby, the fundamental functional potential of glycans is broadly realized, giving ample reason for the interest to delineate detailed structure-function relationships.

With regard to $\mathrm{N}$-glycans, distinct substitutions in the common core region are known from structural glycomics (Wilson et al., 2009; Zuber and Roth, 2009). Their presence can definitely not be dismissed as a random event. As an initial measure to appraise the role of modifications it can give the synthetic route to mature $N$ glycans a clear direction (Brockhausen and Schachter, 1997). As an illuminating example for this role, the relative timing of adding a bisecting $N$-acetylglucosaminyl (GlcNAc) moiety by $\mathrm{N}$-acetylglucosaminyltransferase-III (GnT-III) commits $N$-glycan synthesis either to hybrid or to complex-type $N$-glycans (Brockhausen and Schachter, 1997). Also, its insertion precludes forming branches by GnT-IV/N and the $\alpha 1,6$-fucosylation of the core by fucosyltransferase-VIII (FucT-VIII), the latter substitution mode establishing a second common modification of the core 
$\mathrm{N}$-glycan and a means to inhibit de- $\mathrm{N}$-glycosylation by $N$-glycanase (Suzuki et al., 1997; Ma et al., 2006; Wilson et al., 2009; Zuber and Roth, 2009). Figure 1 shows the precise positions and linkage modes of both mentioned types of covalent addition to the $\mathrm{N}$-glycan core. Having looked at synthesis and cleavage, it becomes an obvious question as to whether the introduction of these two core modifications will affect the properties of the mature $\mathrm{N}$-glycan. That being said, it is intuitively attractive to propose that they might act as molecular switches for the conformational behavior of glycans. Explicitly, the topology of individual glycan antennae can then be altered, and can even connote to modulate lectin binding at branch ends. In this case, core substitutions would qualify as switches with effects in the immediate vicinity, making their presence felt at distant sites.

In what follows we address this issue by reviewing recent results using molecular modeling in silico and cellbinding assays in vitro as well as monitoring biodistribution with neoglycoproteins in vivo. These laboratory tools present synthetic $\mathrm{N}$-glycans on a particular protein backbone after chemical conjugation (Gabius, 2001). In contrast to natural glycoproteins their carbohydrate part is free of microheterogeneity so that neoglycoproteins enable clear-cut delineation of structure-function correlations. The collective evidence establishes a valuable guideline how the natural core substitutions will impinge on serum survival of pharmaglycoproteins. They are amenable to rational manipulation of their carbohydrate part to avoid rapid clearance. The first step to be taken will lead readers from the structure of the $\mathrm{N}$-glycan in two dimensions, as given in Figure 1, to the third dimension of the sugar code.

\section{The third dimension of the sugar code}

A hallmark of oligosaccharides, setting them apart from the highly flexible peptides, is their limited conformational space (von der Lieth et al., 1998). The bulky rings linked by glycosidic bonds can only be accommodated in few positions without clashes. Drawing on the famous lockand-key principle by E. Fischer (1894), 'the carbohydrate moves in solution through a bunch of shapes each of which can be selected by a receptor', thus establishing 'a bunch of keys' (Hardy, 1997). Peptides, in contrast, acquire such conformational preferences by disulfide bonding, in the structural context of a protein or in the presence of a ligand, as shown recently for three $15 \mathrm{mer}$ peptides binding to ganglioside GM1 (Siebert et al., 2006a). That the carbohydrate conformers reside in distinct low-energy positions constitutes the third dimension of the sugar code, the step from a code word written in a sequence to a shape destined for thermodynamically favorable receptor binding (Gabius et al., 2004). The predicted conformer selection upon binding has been widely documented, e.g., in the case of the pentasaccharide of ganglioside GM1 and differential shape recognition of the same sequence by bacterial/human lectins (Siebert et al., 2003). Each energetically privileged conformer ('key') can thus have its distinct bioactivity profile, fitting into a distinct 'lock'.

The principle of limited flexibility does not only hold true for the glycan part directly in contact with receptors. It concerns every glycosidic linkage, and long-range effects are possible in complex glycans. Since conformational preferences in oligovalent $\mathrm{N}$-glycans may therefore have a bearing on the local density of branch-end

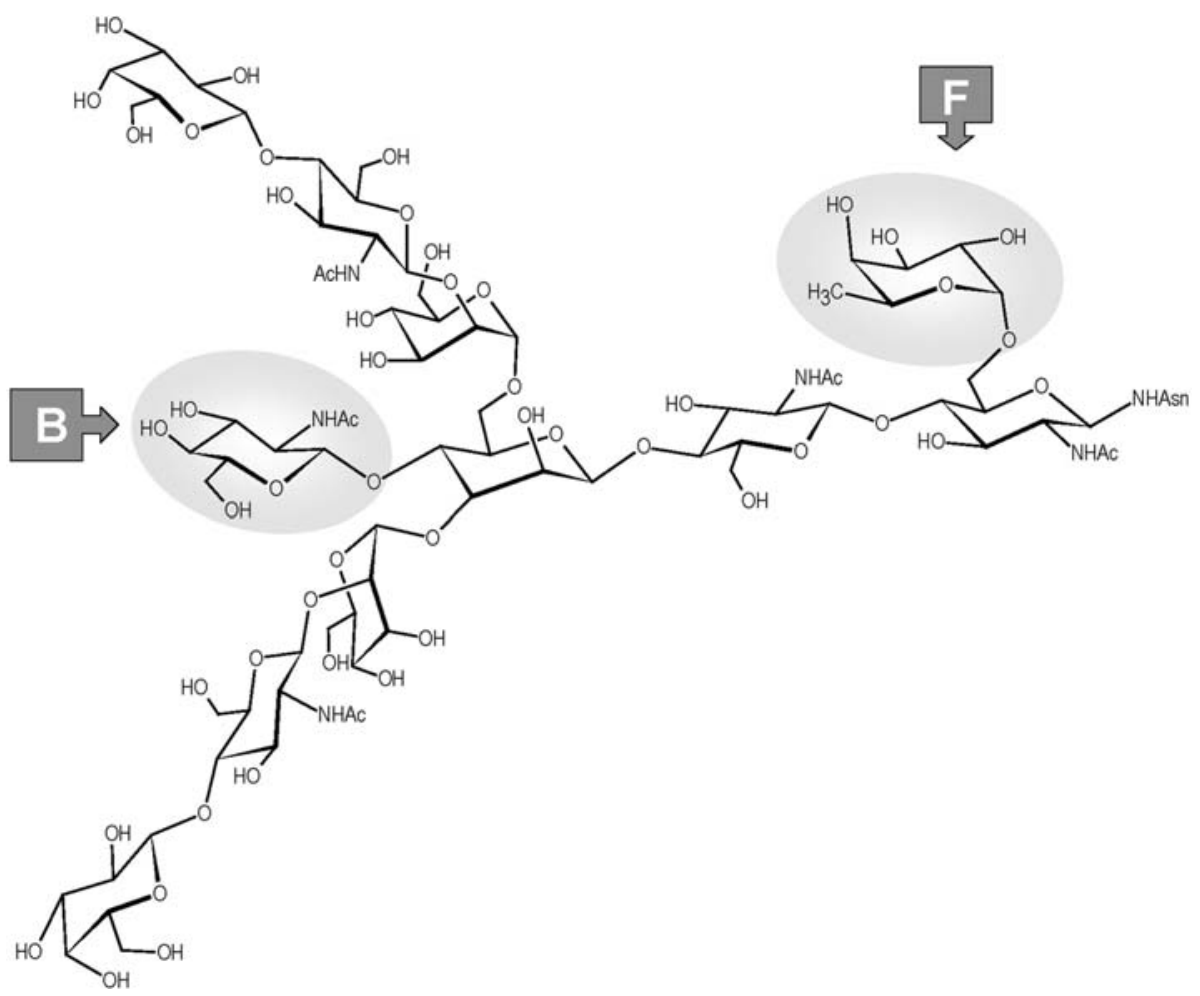

Figure 1 Structure of a biantennary complex-type $N$-glycan (nonasaccharide: Bi9) with the two common core substitutions, i.e., introduction of core-fucose $(F)$ and bisecting GlcNAc (B) moieties, to yield the two monosubstituted decasaccharides BiB10 and $\mathrm{BiF} 10$ and the disubstituted undecasaccharide BiBF11. 

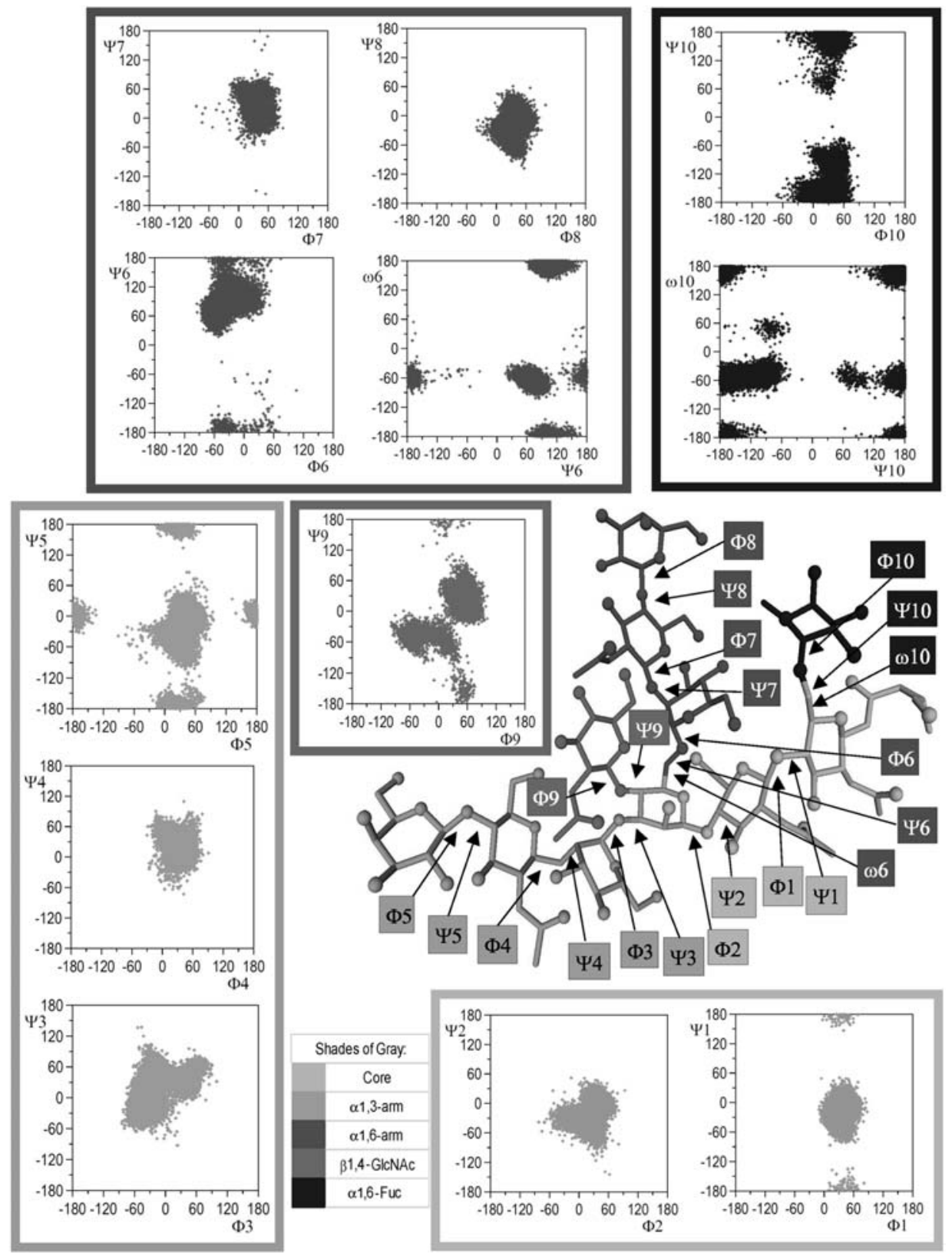

Figure 2 Assignment of symbols to all glycosidic torsion angles for the undecasaccharide BiBF11 using coding in gray tones for clarity (center part).

The conformational flexibility is figured by respective maps in terms of accessible conformational space showing superposed results of three independent molecular dynamics simulations at $400 \mathrm{~K}$ for a period of $1 \mathrm{~ns}$ (peripheral part).

determinants, this factor is supposed to be relevant for modulating avidity of interaction of glycan determinants in natural glycoproteins with lectins. Respective data on importance of branching and local density have already been presented, e.g., in the case of adhesion/growthregulatory mammalian galectins, and are being exploited in glycocluster design (André et al., 1999, 2008; Wu et al., 2004, 2006; Dam et al., 2005; Chabre and Roy, 2009). Having herewith identified shape and local density of branch-end epitopes as salient features in glycan functionality, it is tempting to view core substitutions as molecular switches in this context, and for good reason. Of note, the acquisition of resistance to the plant toxin ricin, specific exclusively for branch-end $\beta$-galactosides, by the LEC10 mutant Chinese hamster ovary $(\mathrm{CHO})$ cells was attributed to induction of GnT-III activity (Campbell and Stanley, 1984; Stanley et al., 1991). The core disubstitution resulting from adding the bisecting GlcNAc residue to core-fucosylated $\mathrm{N}$-glycans of these cells thus appears to be a negative regulator of lectin binding, although ricin will not directly interact with the core section. Computational chemistry is a means to contribute to detect the implied occurrence of spatial long-range effects.

\section{Will core substitutions shift conformational equilibria in $\mathbf{N}$-glycans?}

The hypothesis of switch-like effects on conformational behavior was tested systematically on complex-type 
Bi9

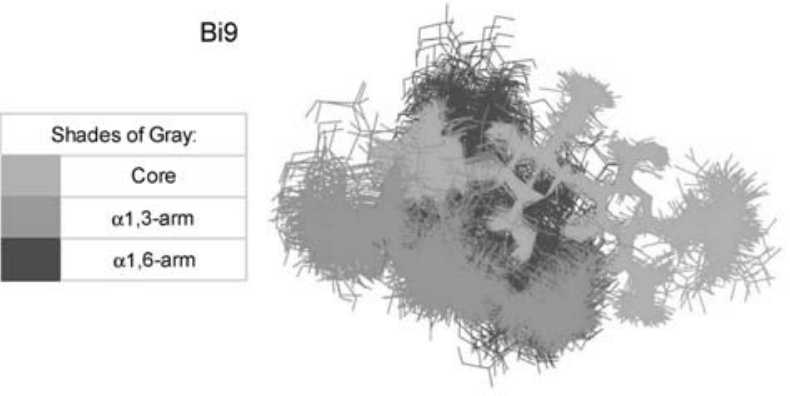

BiB10
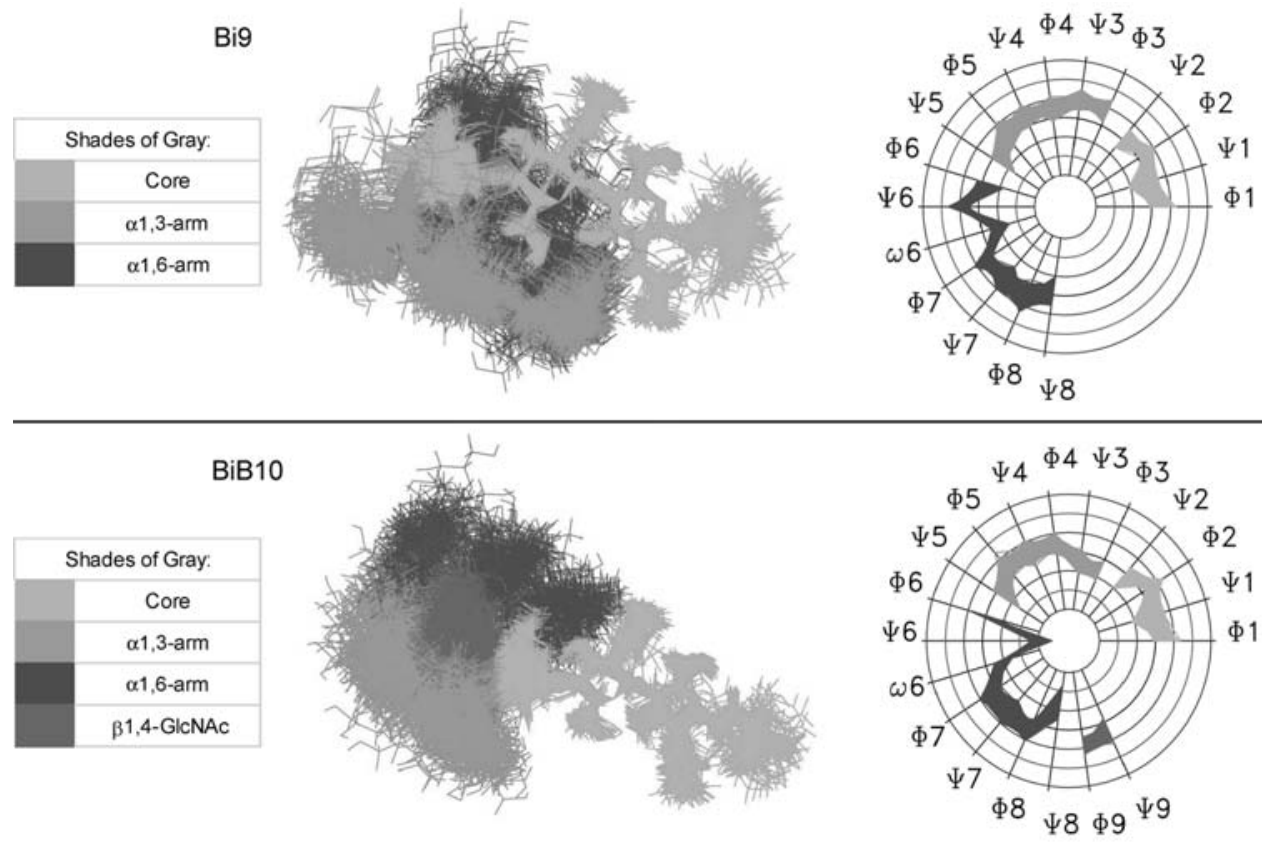

2

\section{1}

BiF10

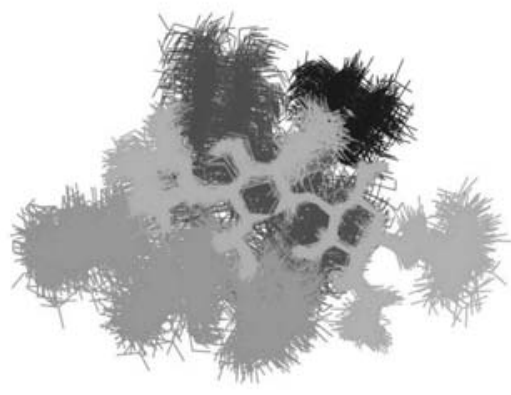

BiBF11
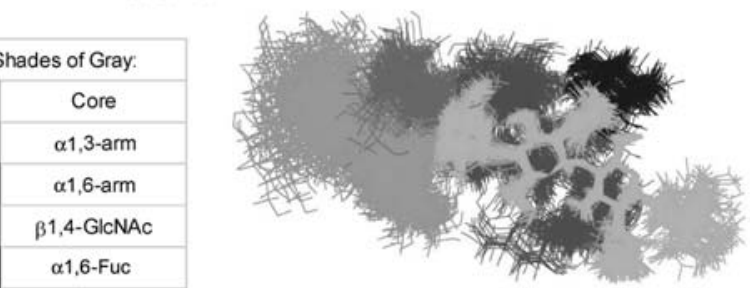
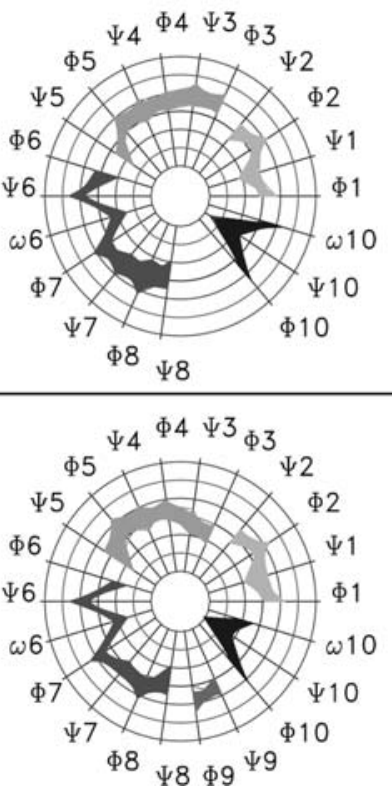

Figure 3 Conformational fingerprint with illustration of the favored spatial constellation of the four $\mathrm{N}$-glycans.

Illustration of energetically privileged conformations from the most densely populated conformational cluster of the $\mathrm{N}$-glycans Bi9 (without core substitution), BiB10/BiF10 (with either bisecting GlcNAc or core-fucose units), and the disubstituted BiBF11 (for structures, please see Figure 1), revealing backfolding of the $\alpha 1,3-a r m$ (Bi9, BiF10) and the $\alpha 1,6$-arm (Bi9, BiF10 and BiBF11) (left panel). Information given in the sets of maps describing intramolecular flexibility at each glycosidic linkage (Figure 2) is transformed into spherical coordinates yielding fingerprint-like representations (right panel). The set of circles in each diagram defines values of torsion angles, starting at $-180^{\circ}$ for the smallest innermost circle and finally reaching $+180^{\circ}$ at the outer boundary. The stepwise change in angle from circle to circle is $60^{\circ}$. In order to maintain cluster continuity a crossing of the line of the innermost circle is allowed to depict values beyond $-180^{\circ}$ in the center in the special case of $\Psi 6$ in the decasaccharide BiB10. Each radial line denotes a particular glycosidic torsion angle, with total numbers of parameters ranging from 17 for the nonasaccharide Bi9 up to 22 for the undecasaccharide BiBF11. For clarity and convenience, the coding in gray tones of Figure 2 is maintained.

biantennary glycans by performing molecular dynamics simulations using TINKER software (Ponder, 2004) with the MM3 parametrization (Stortz, 2005), as described previously (André et al., 2007a). In detail, the conformational space accessed during the simulation periods was compiled for each pair of dihedral glycosidic-linkage angles [for full assignment of symbols to angles in a biantennary complex-type $\mathrm{N}$-glycan (Bi9) with bisecting
GlcNAc (B) and core-fucose (F) moieties, establishing the disubstituted BiBF11 N-glycan, see Figure 2]. As shown, the complete consideration adds up to requirement of analysis of 22 parameters. Their individual monitoring as part of the glycan reveals evident clustering, independently confirmed by 10 ns simulations at $300 \mathrm{~K}$, when inversions of the pyranose rings are precluded, and at $400 \mathrm{~K}$ with three different starting conformations (André 
et al., 2007a). Because the resulting large number of Ramachandran-like conformational maps at each glycosidic angle will inevitably complicate to gain a clear grasp on mutual dependence of conformational transitions in $\alpha 1,3 / 6$-arms, we devised a single-plot fingerprint-like presentation in polar coordinates for each regional portion of the oligosaccharide. To track down an effect of the core substitutions, we start the documentation with the unmodified biantennary glycan (Bi9), then proceeding to the two single-substituted $\mathrm{N}$-glycans, i.e., with either bisecting GlcNAc (BiB10) or core-fucose (BiF10) moieties, respectively, and finally to the disubstituted $\mathrm{N}$-glycan BiBF11 (Figure 3).

In Figure 3, a graphical illustration of the glycan in the most densely populated conformational cluster is given in each case (Figure 3, left), together with its conformational fingerprint (Figure 3, right). In this plot, each radial line represents a particular torsion angle as defined in Figure 2 . The circles symbolize angle specification in steps of $60^{\circ}$, starting with $-180^{\circ}$ at the inner circle. The spatial positions of the most frequently encountered conformational group based on Boltzmann-energy-dependent clustering among the complete array of topological constellations sampled during the simulation periods are depicted (Figure 3). On average, they are rather similar for the two $\mathrm{N}$-glycans Bi9 and BiF10, whereas BiB10 showed altered behavior at the first glycosidic linkages of the $\alpha 1,3 / 6$-arms (see especially $\Psi 6$ in the right panel of Figure 3). Importantly, presence of both substitutions in the BiBF11 $\mathrm{N}$-glycan led to a unique distribution of predominantly accessed space. It is characterized by preference to opposite orientations of the $\alpha 1,6$-antenna relative to BiB10 and of the $\alpha 1,3$-antenna relative to Bi9/ BiF10 (Figure 3). In terms of local density of branch-end epitopes, the two galactose residues are on average $5.9 \AA$ apart in the Bi9/BiF10 N-glycans, 8.1 $\AA$ in the BiB10 compound and 22.1 $\AA$ in the BiBF11 glycan (André et al., 2007a). Although flexibility in the $\alpha 1,3 / 6$-linkages extending from the trimannoside part of the core will certainly still enable a dynamic equilibrium between extended and backfolded orientations of the antennae, these calculations nonetheless reveal an indication for shifts in conformational equilibria. They depend on the presence of these two core substitutions. Thus, they are modulators for the shape of $\mathrm{N}$-glycans, and these alterations engender differences in relative positioning of branch-end determinants, as shown in Figure 3 (left) and noted above. As consequence, the reactivity of the glycans to lectins may be subject to changes. If pure glycans were available, this issue could be resolved. In that case, their chemical attachment to a carrier protein will produce the equivalent of natural glycoproteins, a neoglycoprotein, with custom-made ligand part for lectin interaction (Gabius et al., 1988, 1993; Danguy et al., 1994, 1995; Lee and Lee, 1994; Legendre et al., 2004). Toward this end, chemoenzymatic $\mathrm{N}$-glycan synthesis and conjugation to serum albumin as standard carrier was performed in all cases. Biopharmaceutical relevance was attained by monitoring binding of the neoglycoproteins to tumor cells in vitro and biodistribution in vivo. These experiments explore the perspective for lectin-mediated drug targeting and tumor imaging by rational glycoengineering (Yamazaki et al., 2000).

\section{Will core substitutions modulate cell binding?}

Tumor cells of different histogenetic origin were tested with the set of neoglycoproteins under identical experimental conditions (André et al., 1997, 2004, 2007a; Unverzagt et al., 2002). A dependence of carbohydratemediated cell binding on the structural parameters of the glycan chain is evident (Figure 4). Naturally, binding of these glycans to cell surfaces can yet involve the branchend epitopes and also the core region, complicating the interpretation. Both long-range effects and direct participation of the added residue in binding and combinations thereof are possible. Indeed, plant lectins, such as the leguminous agglutinins LCA/PSA or the erythroagglutinating phytohemagglutinin, respectively, serve as examples of lectins homing in on a certain type of core substitution as part of their contact site (Goldstein and Poretz, 1986). In order to relate spatial presentation of galactose residues to cell binding with less ambiguity, we next analyzed $N$-glycan functionality for hepatic uptake in vivo, because 'the rapidity of this transfer of asialoceruloplasmin from plasma to liver has been shown to be dependent upon the integrity of the exposed, terminal
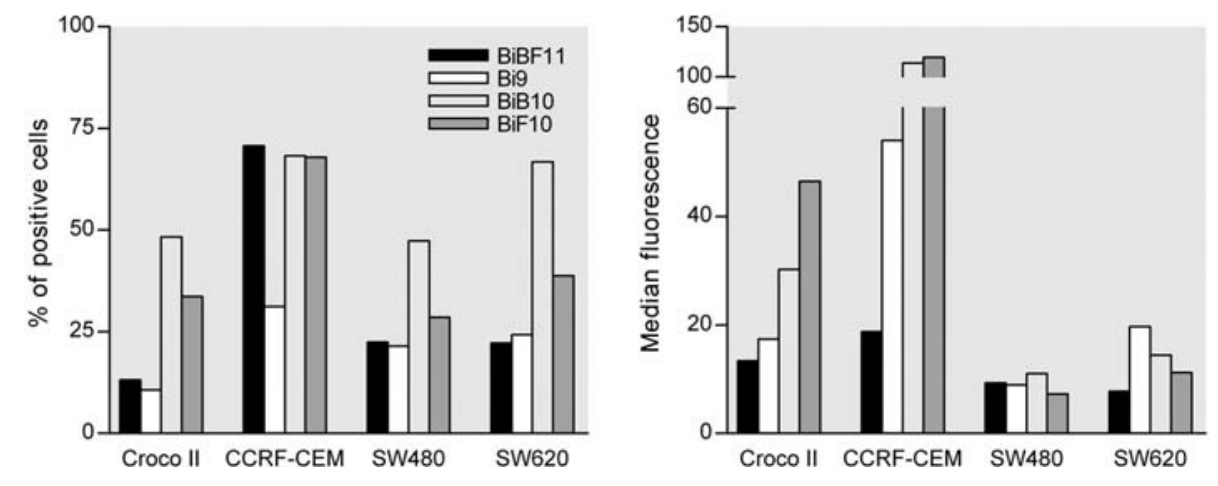

Figure 4 Cell binding of neoglycoproteins in relation to the core structure.

Comparison of fluorescent cell staining (left panel: percentage of positive cells; right panel: median fluorescence intensity) by labeled neoglycoproteins presenting one of the four biantennary complex-type $N$-glycans Bi9, BiB10, BiF10, and BiBF11 (for nomenclature, please see legend to Figure 1) for four human tumor lines: the B-lymphoblastoid line Croco II, the T-lymphoblastoid line CCRF-CEM, and the two colon adenocarcinoma lines SW480/SW620. Standard deviations were generally less than $7.5 \%$. 
galactosyl residues' (Morell et al., 1968). In fact, the socalled asialoglycoprotein receptor of parenchymal liver cells has become a role model for carbohydrate-specific endocytic uptake (Rogers and Kornfeld, 1971; Lee and Lee, 1994; Yamazaki et al., 2000; Park et al., 2005). Monitoring biodistribution of neoglycoproteins, especially blood and liver contents, is thus a sensitive diagnostic tool to recognize an influence of $\mathrm{N}$-glycan core substitution on lectin-binding properties, with clinical perspectives. Systematic experimental series with neoglycoproteins presenting the different $N$-glycans have thus been carried out (André et al., 1997, 2004, 2007a; Unverzagt et al., 2002).

As shown in Figure 5, marked changes are detectable. Most prominently, presence of both core substitutions prolongs serum survival (Figure 5, top). The experiments in tumor-bearing mice also made possible assessment of the potential of the neoglycoproteins for imaging. Of note in this respect, the introduction of a third branch by a $\beta 1,2$-addition of GIcNAc to the central mannose unit in the core-fucosylated $\mathrm{N}$-glycan (LEC14 modification) and its galactosylation yielded a favorable tumor/blood ratio (André et al., 2005). The same effect can alternatively be reached by $\alpha 2,3$-sialylation in all four cases, excluding a strong contribution of core substitutions as a major contact point for the hepatic clearance route (Figure 5, middle). This type of addition of sialic acids to the glycan antennae not only introduces a negative charge to the oligosaccharide but also blocks lectin binding in this system (Morell et al., 1968). However, what matters is the mode of sialic acid addition, not its mere presence, an instructive example of the versatility of glycans to obtain non-synonymous signals from isomers (Reuter and Gabius, 1996, 1999). In other words, sialylation in $\alpha 2,3-$ or $\alpha 2,6$-linkages facilitates to generate distinct code words due to characteristic shape and flexibility patterns (Siebert et al., 2006b). In our case, the positional effect of sialylation is revealed by comparatively analyzing the two sets of sialylated $\mathrm{N}$-glycans. Historically, following its discovery the hepatic asialoglycoprotein receptor has been shown to tolerate $\alpha 2,6$-sialylation as branch-end modification, with species-dependent differences (Park and Baenziger, 2004; Park et al., 2005). Marked differences in serum clearance and hepatic content were thus detected between $N$-glycans with $\alpha 2,3-$ or $\alpha 2,6$-sialylation, and an influence of core substitution for $\alpha 2,6$-sialylated $N$-glycans was noted (Figure 5, middle and bottom).

That structural parameters in the $N$-glycan affect lectin reactivity is further underscored by similarly testing the two common isomers of triantennary $N$-glycans. The two branching modes are illustrated in Figure 6 as fully protected octasaccharides. They were the starting point for processing by deprotection and the enzymatic chain elongation (André et al., 2006). Reflecting the binding of the $\beta 1,4$-linked branch of the $\alpha 1,3$-arm to the minor subunits of the hepatic C-type lectin and the rapid clearance of the glycoprotein asialofetuin harboring type I branching in the triantennary $\mathrm{N}$-glycan chains (Lee et al., 1983; Kojima et al., 1990; Rice et al., 1990), hepatic contents were different between the isomers despite their identical number of terminal galactose moieties (Figure
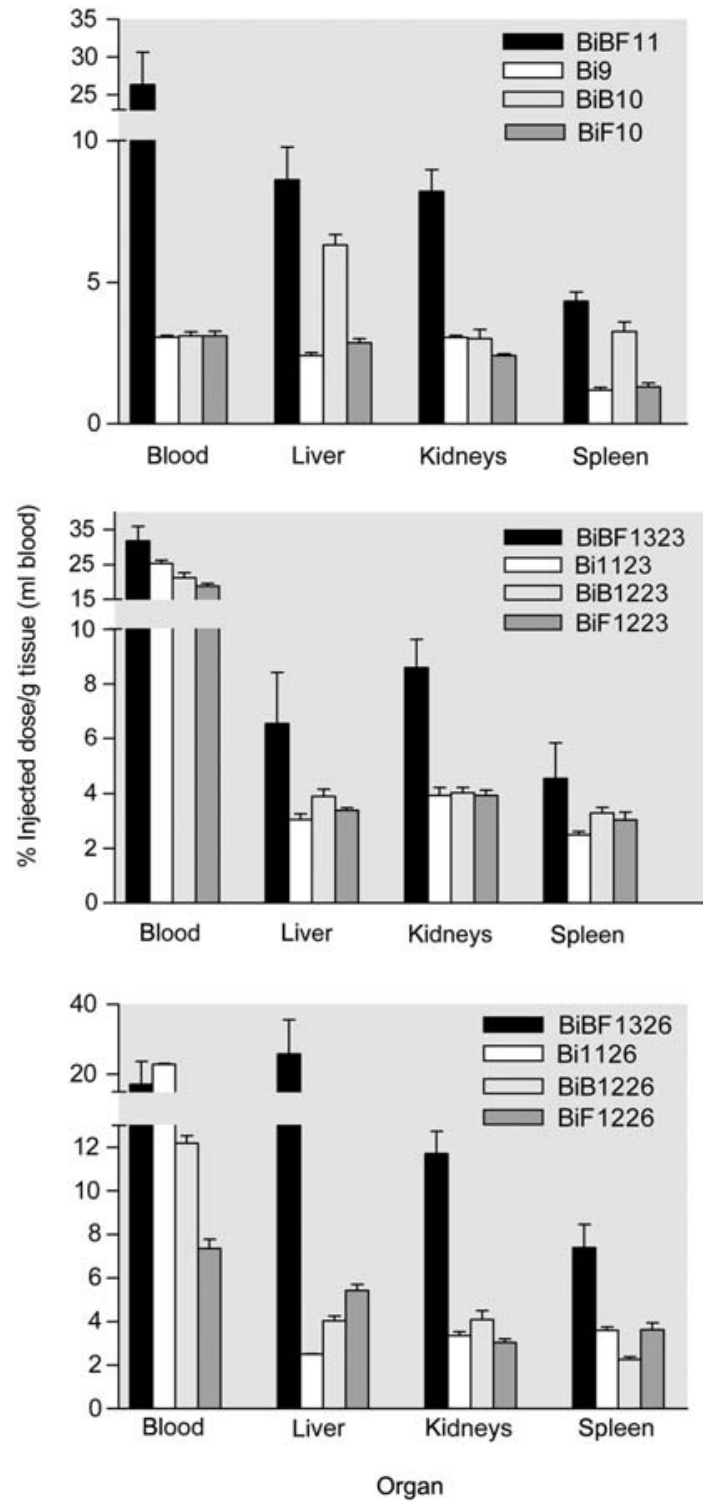

Figure 5 Comparison of $\mathrm{N}$-glycan-type-dependent biodistribution profiles of radioiodinated neoglycoproteins $1 \mathrm{~h}$ after their intravenous injection into tumor-bearing mice.

Datasets for the four types of sialic acid-free $\mathrm{N}$-glycans are presented in the top panel, for the set of fully $\alpha 2,3$-sialylated $\mathrm{N}$-glycans in the middle panel, and for fully $\alpha 2,6$-sialylated $N$ glycans in the bottom panel (for definition of glycan acronyms, please see legend to Figure 1).

7). This case set another example for differences in bioactivity by glycan tailoring. With potential for tumor delivery, uptake at this site was rather high with $4.18 \pm 0.5 \%$ of the injected dose after $1 \mathrm{~h}$ for the $\alpha 2,3$-sialylated second isoform (André et al., 2006). It exceeded that into any organ. In conclusion, these results establish a guideline for rational glycoengineering, which can even prompt to envision devising unnatural substitutions.

\section{Concluding remarks}

The impact of posttranslational modifications, such as methylation or phosphorylation, on protein functionality is well documented and widely appreciated. The growing 

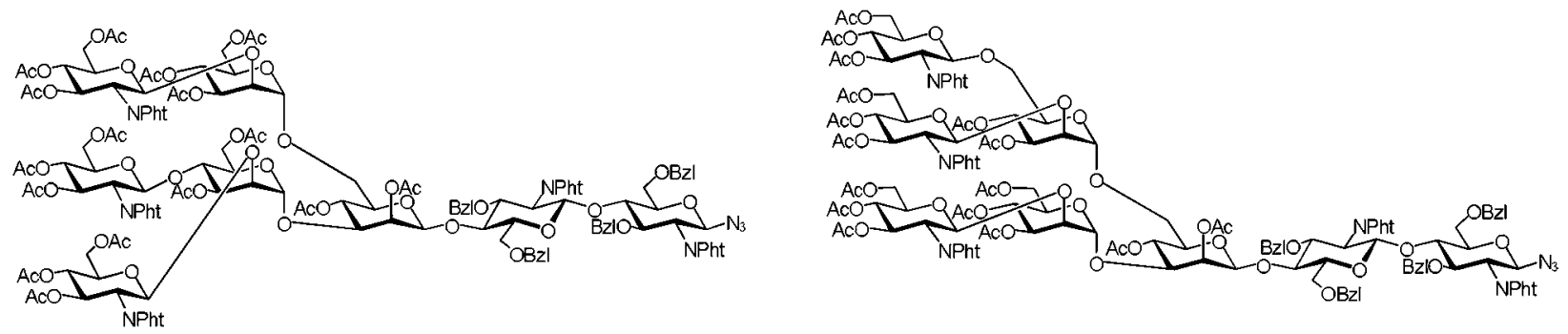

Figure 6 Structures of the two fully protected, chemically synthesized octasaccharides.

Indicated are the two branching modes of triantennary complex-type $N$-glycans with type I branching composed of $\beta 1,2 / \beta 1,4-$ antennae in the $\alpha 1,3$-arm (right) and type II branching with $\beta 1,2 / \beta 1,6$-antennae in the $\alpha 1,6$-arm (left).

awareness of the exceptional talents of glycans in information coding is the driving force to keep the body of evidence for the assumed 'discrete recognitional role' (Winterburn and Phelps, 1972) of the glycan part of cellular glycoconjugates dynamically growing. This concept for a sugar code leads to the notion that substitutions in glycan chains alter the nature of the conveyed signal in functional terms. On the contrary, a provocative view would attribute their occurrence to random events. Here, we focused on two common core substitutions of $\mathrm{N}$-glycans, addressing the issues whether their presence can modulate conformational and lectin-binding properties. The results presented herein reveal the nature of corefucose and bisecting GlcNAc units as molecular switches altering the accessed conformational space of the N-glycan by long-range effects. These changes can help rationalize the measured impact of presence of substitution on glycoproteins, such as growth factor receptors when harboring core fucosylation (Wang et al., 2006; Matsumoto et al., 2008; Honke and Taniguchi, 2009), and on the lectin-binding properties of glycans. The presented biodistribution data, also covering isomers of triantennary $\mathrm{N}$-glycans, provide a guideline for rational glycoengineering of glycosylated pharmaproteins. Owing to the potency of tissue lectins as elicitors of signaling in

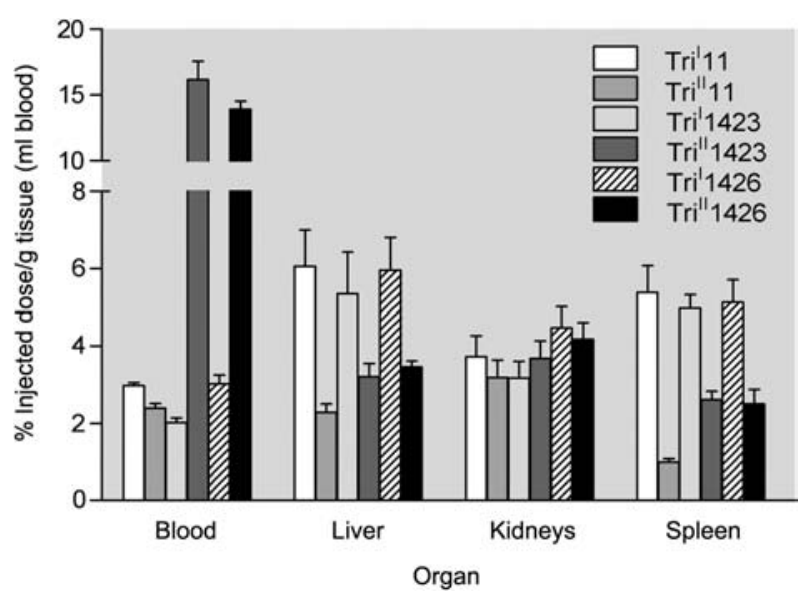

Figure 7 Comparison of $\mathrm{N}$-glycan-type-dependent biodistribution profiles of radioiodinated neoglycoproteins $1 \mathrm{~h}$ after their intravenous injection into tumor-bearing mice.

Data are given for the sialic acid-free undecasaccharides with type I/II branching Tri'11/Tril11 (for structures of the two forms differing in branching mode, see Figure 6), the fully $\alpha 2,3$-sialylated tetradecasaccharides Tri'1423/Tri"1423, and the fully $\alpha 2,6-$ sialylated tetradecasaccharides Tri'1426/Tril"1426. concert with particular glycoprotein targets, such as the $\alpha_{5} \beta_{1}$-integrin or CD7 or as glycoprotein-specific transport vehicles (Rappl et al., 2002; Villalobo et al., 2006; André et al., 2007b; Morelle et al., 2009), even a low overall frequency of physiological substitution hitting these functionally important sites will be effective. Structural changes mapped by glycophenotyping, e.g., by glycoproteomics as a current approach in the quest to define new cancer biomarkers, can thus hereby reach a functional meaning. Finally, the unraveled activity of these $N$ glycan core substitutions can be emblematic for other types of glycan substitutions, e.g., when adorning $N$-glycan cores with $\alpha 1,3$-linked fucose or $\beta 1,2$-linked xylose units in plants and insects or with further GlcNAc moieties in $\mathrm{CHO}$ glycosylation mutants or when leading to the different cores of mucin-type O-glycans and branching in O-mannosylated glycans (Patnaik and Stanley, 2006; Patsos and Corfield, 2009; Wilson et al., 2009).

\section{Acknowledgments}

We gratefully acknowledge Drs. B. Friday, G. Ippans, S. Namirha, W. Notelecs, and W. Tab for inspiring discussions as well as an EC Marie Curie Research Training Network (contract no. MCRTN-CT-2005-19561), the VEGA Slovak grant agency (contract no. 2/7053/07), the research initiative LMUexcellent and the Verein zur Förderung des biologisch-technologischen Fortschritts in der Medizin e.V. for generous financial support.

\section{References}

André, S., Unverzagt, C., Kojima, S., Dong, X., Fink, C., Kayser, K., and Gabius, H.-J. (1997). Neoglycoproteins with the synthetic complex biantennary nonasaccharide or its $\alpha 2,3 /$ $\alpha 2,6$-sialylated derivatives: their preparation, assessment of their ligand properties for purified lectins, for tumor cells in vitro, and in tissue sections, and their biodistribution in tumor-bearing mice. Bioconj. Chem. 8, 845-855.

André, S., Ortega, P.J., Perez, M.A., Roy, R., and Gabius, H.-J. (1999). Lactose-containing starburst dendrimers: influence of dendrimer generation and binding-site orientation of receptors (plant/animal lectins and immunoglobulins) on binding properties. Glycobiology 9, 1253-1261.

André, S., Unverzagt, C., Kojima, S., Frank, M., Seifert, J., Fink, C., Kayser, K., von der Lieth, C.-W., and Gabius, H.-J. (2004). Determination of modulation of ligand properties of synthetic complex-type biantennary $\mathrm{N}$-glycans by introduction of bisecting GlcNAc in silico, in vitro and in vivo. Eur. J. Biochem. 271, 118-134. 
André, S., Kojima, S., Prahl, I., Lensch, M., Unverzagt, C., and Gabius, H.-J. (2005). Introduction of extended LEC14-type branching into core-fucosylated biantennary N-glycan. Glycoengineering for enhanced cell binding and serum clearance of the neoglycoprotein. FEBS J. 272, 1986-1998.

André, S., Kojima, S., Gundel, G., Russwurm, R., Schratt, X., Unverzagt, C., and Gabius, H.-J. (2006). Branching mode in complex-type triantennary $\mathrm{N}$-glycans as regulatory element of their ligand properties. Biochim. Biophys. Acta 1760, 768-782.

André, S., Kožár, T., Schuberth, R., Unverzagt, C., Kojima, S., and Gabius, H.-J. (2007a). Substitutions in the N-glycan core as regulators of biorecognition: the case of core-fucose and bisecting GlcNAc moieties. Biochemistry 46, 6984-6995.

André, S., Sanchez-Ruderisch, H., Nakagawa, H., Buchholz, M., Kopitz, J., Forberich, P., Kemmner, W., Böck, C., Deguchi, K., Detjen, K.M., et al. (2007b). Tumor suppressor p16 ink4a: modulator of glycomic profile and galectin-1 expression to increase susceptibility to carbohydrate-dependent induction of anoikis in pancreatic carcinoma cells. FEBS J. 274, 3233-3256.

André, S., Sansone, F., Kaltner, H., Casnati, A., Kopitz, J., Gabius, H.-J., and Ungaro, R. (2008). Calix[n]arene-based glycoclusters: bioactivity of thiourea-linked galactose/lactose moieties as inhibitors of binding of medically relevant lectins to a glycoprotein and cell-surface glycoconjugates and selectivity among human adhesion/growth-regulatory galectins. ChemBioChem 9, 1649-1661.

Barbieri, M., ed. (2008). The Codes of Life. The Rules of Macroevolution (Heidelberg/New York: Springer).

Brockhausen, I. and Schachter, H. (1997). Glycosyltransferases involved in N- and O-glycan biosynthesis. In: Glycosciences: Status and Perspectives, H.-J. Gabius and S. Gabius, eds. (Weinheim, Germany: Chapman and Hall), pp. 79-113.

Campbell, C. and Stanley, P. (1984). A dominant mutation to ricin resistance in Chinese hamster ovary cells induces UDP-GIcNAc:glycopeptide $\beta-4-N$-acetylglucosaminyltransferase III activity. J. Biol. Chem. 259, 13370-13378.

Chabre, Y. and Roy, R. (2009). Chemist's way to prepare multivalency. In: The Sugar Code. Fundamentals of Glycosciences, H.-J. Gabius, ed. (Weinheim, Germany: Wiley$\mathrm{VCH}$ ), in press.

Dam, T.K., Gabius, H.-J., André, S., Kaltner, H., Lensch, M., and Brewer, C.F. (2005). Galectins bind to the multivalent glycoprotein asialofetuin with enhanced affinities and a gradient of decreasing binding constants. Biochemistry 44, 1256412571.

Danguy, A., Akif, F., Pajak, B., and Gabius, H.-J. (1994). Contribution of carbohydrate histochemistry to glycobiology. Histol. Histopathol. 9, 155-171.

Danguy, A., Kayser, K., Bovin, N.V., and Gabius, H.-J. (1995). The relevance of neoglycoconjugates for histology and pathology. Trends Glycosci. Glycotechnol. 7, 261-275.

Fischer, E. (1894). Einfluß der Configuration auf die Wirkung der Enzyme. Ber. Dt. Chem. Ges. 27, 2985-2993.

Gabius, H.-J. (2001) Glycohistochemistry: the why and how of detection and localization of endogenous lectins. Anat. Histol. Embryol. 30, 3-31.

Gabius, H.-J., ed. (2009). The Sugar Code. Fundamentals of Glycosciences (Weinheim, Germany: Wiley-VCH).

Gabius, H.-J., Bodanowitz, S., and Schauer, A. (1988). Endogenous sugar-binding proteins in human breast tissue and benign and malignant breast lesions. Cancer 61, 1125-1131.

Gabius, H.-J., Gabius, S., Zemlyanukhina, T.V., Bovin, N.V., Brinck, U., Danguy, A., Joshi, S.S., Kayser, K., Schottelius, J., Sinowatz, F., et al. (1993). Reverse lectin histochemistry: design and application of glycoligands for detection of cell and tissue lectins. Histol. Histopathol. 8, 369-383.

Gabius, H.-J., Siebert, H.-C., André, S., Jiménez-Barbero, J., and Rüdiger, H. (2004). Chemical biology of the sugar code. ChemBioChem 5, 740-764.
Goldstein, I.J. and Poretz, R.D. (1986). Isolation, physicochemical characterization, and carbohydrate-binding specificity of lectins. In: The Lectins. Properties, Functions, and Applications in Biology and Medicine, I.E. Liener, N. Sharon and I.J. Goldstein, eds. (San Diego, CA, USA: Academic Press), pp. 33-247.

Hardy, B.J. (1997). The glycosidic linkage flexibility and timescale similarity hypothesis. J. Mol. Struct. 395-396, 187200

Honke, K. and Taniguchi, N. (2009). Animal models to delinate glycan functionality. In: The Sugar Code. Fundamentals of Glycosciences, H.-J. Gabius, ed. (Weinheim, Germany: Wiley-VCH), in press.

Kojima, S., Ishido, M., Kubota, K., Kubodera, A., Hellmann, T., Kohnke-Godt, B., Wosgien, B., and Gabius, H.-J. (1990). Tissue distribution of radioiodinated neoglycoproteins and mammalian lectins. Biol. Chem. Hoppe-Seyler 371, 331-338.

Laine, R.A. (1997). The information-storing potential of the sugar code. In: Glycosciences: Status and Perspectives, H.-J. Gabius and S. Gabius, eds. (Weinheim, Germany: Chapman and Hall), pp. 1-14.

Lee, R.T. and Lee, Y.C. (1994). Enhanced biochemical affinities of multivalent neoglycoconjugates. In: Neoglycoconjugates. Preparation and Applications, Y.C. Lee and R.T. Lee, eds. (San Diego, CA, USA: Academic Press), pp. 23-50.

Lee, Y.C., Townsend, R.R., Hardy, M.R., Lönngren, J., Arnarp, J., Haraldsson, M., and Lönn, H. (1983). Binding of synthetic oligosaccharides to the hepatic Gal/GalNAc lectin. Dependence on fine structural features. J. Biol. Chem. 258, 199-202.

Legendre, H., Decaestecker, C., Gbenou, M.G., Nagy, N., Hendlisz, A., André, S., Pector, J.C., Kiss, R., and Gabius, H.-J. (2004). Prognostic stratification of Dukes B colon cancer by a neoglycoprotein. Int. J. Oncol. 25, 269-276.

Ma, B., Simala-Grant, J.L., and Taylor, D.E. (2006). Fucosylation in prokaryotes and eukaryotes. Glycobiology 16, 158R184R.

Matsumoto, K., Yokote, H., Arao, T., Maegawa, M., Tanaka, K., Fujita, Y., Shimizu, C., Hanafusa, T., Fujiwara, Y., and Nishio, K. (2008). N-Glycan fucosylation of epidermal growth factor receptor modulates receptor activity and sensitivity to epidermal growth factor receptor tyrosine kinase inhibitor. Cancer Sci. 99, 1611-1617.

Morell, A.G., Irvine, R.A., Sternlieb, I., Scheinberg, I.H., and Ashwell, G. (1968). Physical and chemical studies on ceruloplasmin. V. Metabolic studies on sialic acid-free ceruloplasmin in vivo. J. Biol. Chem. 243, 155-159.

Morelle, W., Stechly, L., André, S., Van Seuningen, I., Porchet, N., Gabius, H.-J., Michalski, J.-C., and Huet, G. (2009). Glycosylation pattern of brush border-associated glycoproteins in enterocyte-like cells: involvement of complex-type N-glycans in apical trafficking. Biol. Chem. 390, 529-544.

Park, E.I. and Baenziger, J.U. (2004). Closely related mammals have distinct asialoglycoprotein receptor carbohydrate specificities. J. Biol. Chem. 279, 40954-40959.

Park, E.I., Mi, Y., Unverzagt, C., Gabius, H.-J., and Baenziger, J.U. (2005). The asialoglycoprotein receptor clears glycoconjugates terminating with sialic acid $\alpha 2,6$ GalNAc. Proc. Natl. Acad. Sci. USA 102, 17125-17129.

Patnaik, S.K. and Stanley, P. (2006). Lectin-resistant CHO glycosylation mutants. Methods Enzymol. 416, 159-182.

Patsos, G. and Corfield, A. (2009). O-Glycosylation: structural diversity and functions. In: The Sugar Code. Fundamentals of Glycosciences, H.-J. Gabius, ed. (Weinheim, Germany: Wiley- $\mathrm{VCH}$ ), in press.

Ponder, J.W. (2004). User's Guide for TINKER Version 4.2 (St. Louis, MO, USA: Washington University School of Medicine).

Rappl, G., Abken, H., Muche, J.M., Sterry, W., Tilgen, W., André, S., Kaltner, H., Ugurel, S., Gabius, H.-J., and Reinhold, U. (2002). CD4 ${ }^{+}$CD7- leukemic T cells from patients with Sézary syndrome are protected from galectin-1-triggered $\mathrm{T}$ cell death. Leukemia 16, 840-845. 
Reuter, G. and Gabius, H.-J. (1996). Sialic acids. Structureanalysis-metabolism-occurrence-recognition. Biol. Chem. Hoppe-Seyler 377, 325-342.

Reuter, G. and Gabius, H.-J. (1999). Eukaryotic glycosylation: whim of nature or multipurpose tool? Cell. Mol. Life Sci. 55, 368-422.

Rice, K.G., Weisz, O.A., Barthel, T., Lee, R.T., and Lee, Y.C. (1990). Defined geometry of binding between triantennary glycopeptide and the asialoglycoprotein receptor of rat heptocytes. J. Biol. Chem. 265, 18429-18434.

Rogers, J.C. and Kornfeld, S. (1971). Hepatic uptake of proteins coupled to fetuin glycopeptide. Biochem. Biophys. Res. Commun. 45, 622-629.

Sharon, N. and Lis, H. (1997). Glycoproteins: structure and function. In: Glycosciences: Status and Perspectives, H.-J. Gabius and S. Gabius, eds. (Weinheim, Germany: Chapman and Hall), pp. 133-162.

Siebert, H.-C., André, S., Lu, S.-Y., Frank, M., Kaltner, H., van Kuik, J.A., Korchagina, E.Y., Bovin, N.V., Tajkhorshid, E., Kaptein, R., et al. (2003). Unique conformer selection of human growth-regulatory lectin galectin-1 for ganglioside $\mathrm{GM}_{1}$ versus bacterial toxins. Biochemistry 42, 14762-14773.

Siebert, H.-C., Born, K., André, S., Frank, M., Kaltner, H., von der Lieth, C.-W., Heck, A.J.R., Jiménez-Barbero, J., Kopitz, J., and Gabius, H.-J. (2006a). Carbohydrate chain of ganglioside $\mathrm{GM}_{1}$ as a ligand: identification of the binding strategies of three 15 mer peptides and their divergence from the binding modes of growth-regulatory galectin-1 and cholera toxin. Chem. Eur. J. 12, 388-402.

Siebert, H.-C., Rosen, J., Seyrek, K., Kaltner, H., André, S., Bovin, N.V., Nyholm, P.-G., Sinowatz, F., and Gabius, H.-J. (2006b). $\alpha 2,3 / \alpha 2,6$-Sialylation of N-glycans: non-synonymous signals with marked developmental regulation in bovine reproductive tracts. Biochimie 88, 399-410.

Sims, R.J. III and Reinberg, D. (2008). Is there a code embedded in proteins that is based on post-translational modifications? Nat. Rev. Mol. Cell Biol. 9, 1-6.

Solís, D., Jiménez-Barbero, J., Kaltner, H., Romero, A., Siebert, H.-C., von der Lieth, C.-W., and Gabius, H.-J. (2001). Towards defining the role of glycans as hardware in information storage and transfer: basic principles, experimental approaches and recent progress. Cells Tiss. Organs 168, 5-23.

Stanley, P., Sundaram, S., and Sallustio, S. (1991). A subclass of cell surface carbohydrates revealed by a $\mathrm{CHO}$ mutant with two glycosylation mutations. Glycobiology 1, 307-314.

Stortz, C.A. (2005). Comparative performances of MM3(92) and two TINKER MM3 versions for the modeling of carbohydrates. J. Comput. Chem. 26, 471-483.

Suzuki, T., Kitajima, K., Inoue, S., and Inoue, Y. (1997). Occurrence and potential functions of $\mathrm{N}$-glycanases. In: Glycosciences: Status and Perspectives, H.-J. Gabius and S.
Gabius, eds. (Weinheim, Germany: Chapman and Hall), pp. 121-131.

Unverzagt, C., André, S., Seifert, J., Kojima, S., Fink, C., Srikrishna, G., Freeze, H., Kayser, K., and Gabius, H.-J. (2002). Structure-activity profiles of complex biantennary glycans with core fucosylation and with/without additional $\alpha 2,3 / \alpha 2,6$ sialylation: synthesis of neoglycoproteins and their properties in lectin assays, cell binding, and organ uptake. J. Med. Chem. 45, 478-491.

Villalobo, A., Nogales-González, A., and Gabius, H.-J. (2006). A guide to signalling pathways connecting protein-carbohydrate interaction with the emerging versatile effector functionality of mammalian lectins. Trends Glycosci. Glycotechnol. 18, 1-37.

von der Lieth, C.-W., Siebert, H.-C., Kožár, T., Burchert, M., Frank, M., Gilleron, M., Kaltner, H., Kayser, G., Tajkhorshid, E., Bovin, N.V., et al. (1998). Lectin ligands: new insights into their conformations and their dynamic behavior and the discovery of conformer selection by lectins. Acta Anat. 161, 91-109.

Wang, X., Gu, J., Ihara, H., Miyoshi, E., Honke, K., and Taniguchi, N. (2006). Core fucosylation regulates epidermal growth factor receptor-mediated intracellular signaling. J. Biol. Chem. 281, 2572-2577.

Wilson, I.B.H., Paschinger, K., and Rendić, D. (2009). Glycosylation of model and 'lower' organisms. In: The Sugar Code. Fundamentals of Glycosciences, H.-J. Gabius, ed. (Weinheim, Germany: Wiley-VCH), in press.

Winterburn, P.J. and Phelps, C.F. (1972). The significance of glycosylated proteins. Nature 236, 147-151.

Wu, A.M., Wu, J.H., Liu, J.-H., Singh, T., André, S., Kaltner, H., and Gabius, H.-J. (2004). Effects of polyvalency of glycotopes and natural modifications of human blood group $\mathrm{ABH} /$ Lewis sugars at the Gal $\beta 1$-terminated core saccharides on the binding of domain-I of recombinant tandem-repeat-type galectin-4 from rat gastrointestinal tract (G4-N). Biochimie 86, 317-326.

Wu, A.M., Singh, T., Wu, J.H., Lensch, M., André, S., and Gabius, H.-J. (2006). Interaction profile of galectin-5 with free saccharides and mammalian glycoproteins: probing its fine specificity and the effect of naturally clustered ligand presentation. Glycobiology 16, 524-537.

Yamazaki, N., Kojima, S., Bovin, N.V., André, S., Gabius, S., and Gabius, H.-J. (2000). Endogenous lectins as targets for drug delivery. Adv. Drug Deliv. Rev. 43, 225-244.

Zuber, C. and Roth, J. (2009). N-Glycosylation. In: The Sugar Code. Fundamentals of Glycosciences, H.-J. Gabius, ed. (Weinheim, Germany: Wiley-VCH), in press.

Received January 15, 2009; accepted March 31, 2009 